\title{
LAr Dewar Coil Feed Frame Pipe Analysis (Structural Analysis of General Structures, SAGS)
}

\section{D0 ENGINEERING NOTE \\ EN-3740.510-239}

A. W. Parker

January 31,1990 


\section{LAr Dewar Coil Feed, Frame Pipe Analysis}

This frame pipe analysis addresses the D0 LAr dewar Condenser tube (coil) feed pipe thermal contraction stresses and moments. The configuration is shown in PEI drawing C-32545, Rev 0 , and the parameters detailed in the letter of November 20,1989 , located in appendix A.

Note that all other thermal considerations for these condensing coils have been made in DO EN 3740.512-234. The conclusion of this report is the feed lines, previously deemed appropriate without detailed analysis, have been demonstrated to be adequately designed for the intended service and require no further investigation. 


\author{
S A G S \\ STATIC ANALYSIS OF GENERAL STRUCTURES \\ STRUCTURAL DYNAMICS RESEARCH CORPORATION
}

FRAME PIPE ANALYSIS LAR DEWAR COIL FEED $2 / 6 / 90$

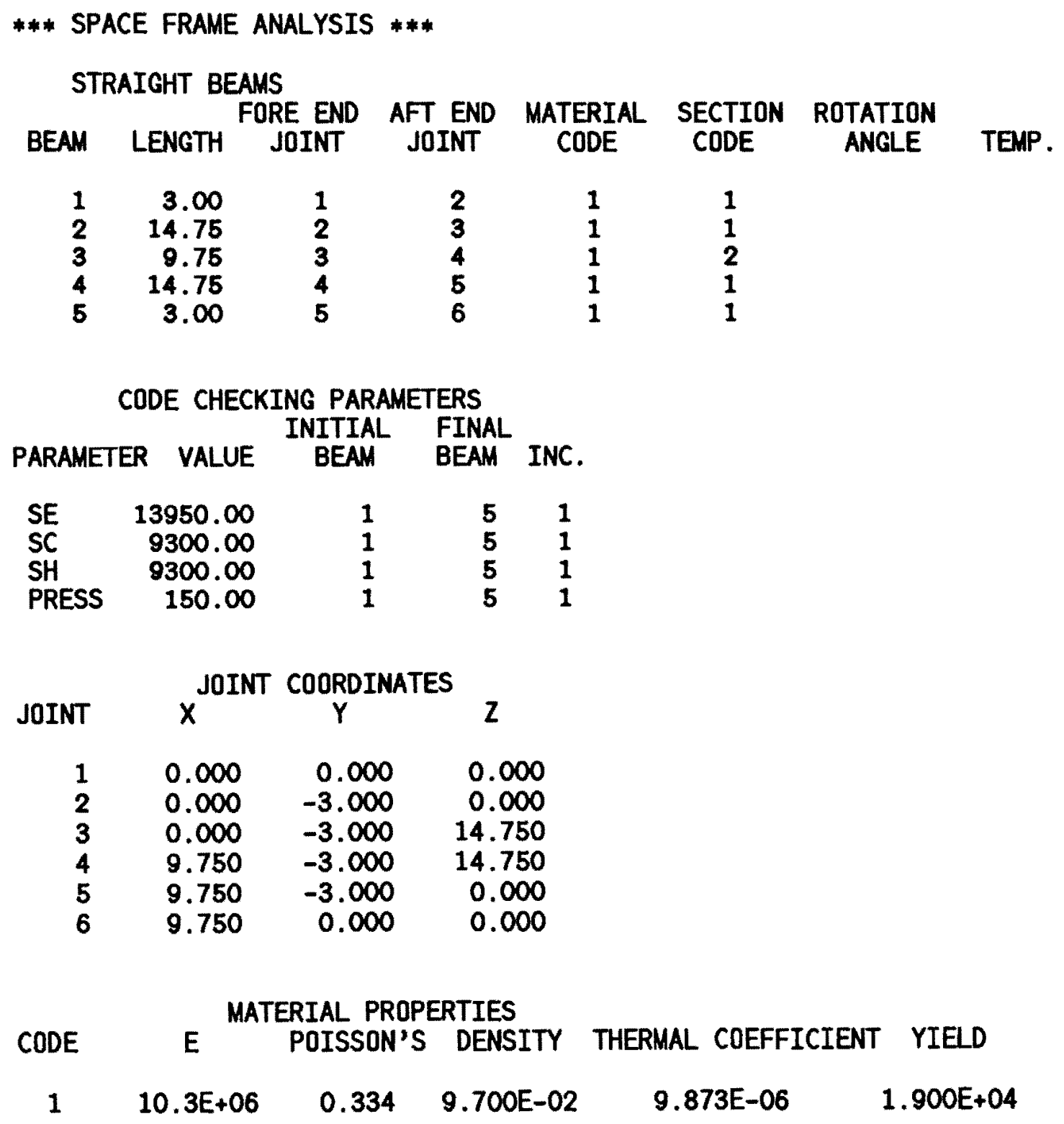


TUESDAY

6-FEB-90

$09: 36: 31$

PAGE 2

STATIC ANALYSIS OF GENERAL STRUCTURES

FRAME PIPE ANALYSIS LAR DEMAR COIL FEED 2/6/90

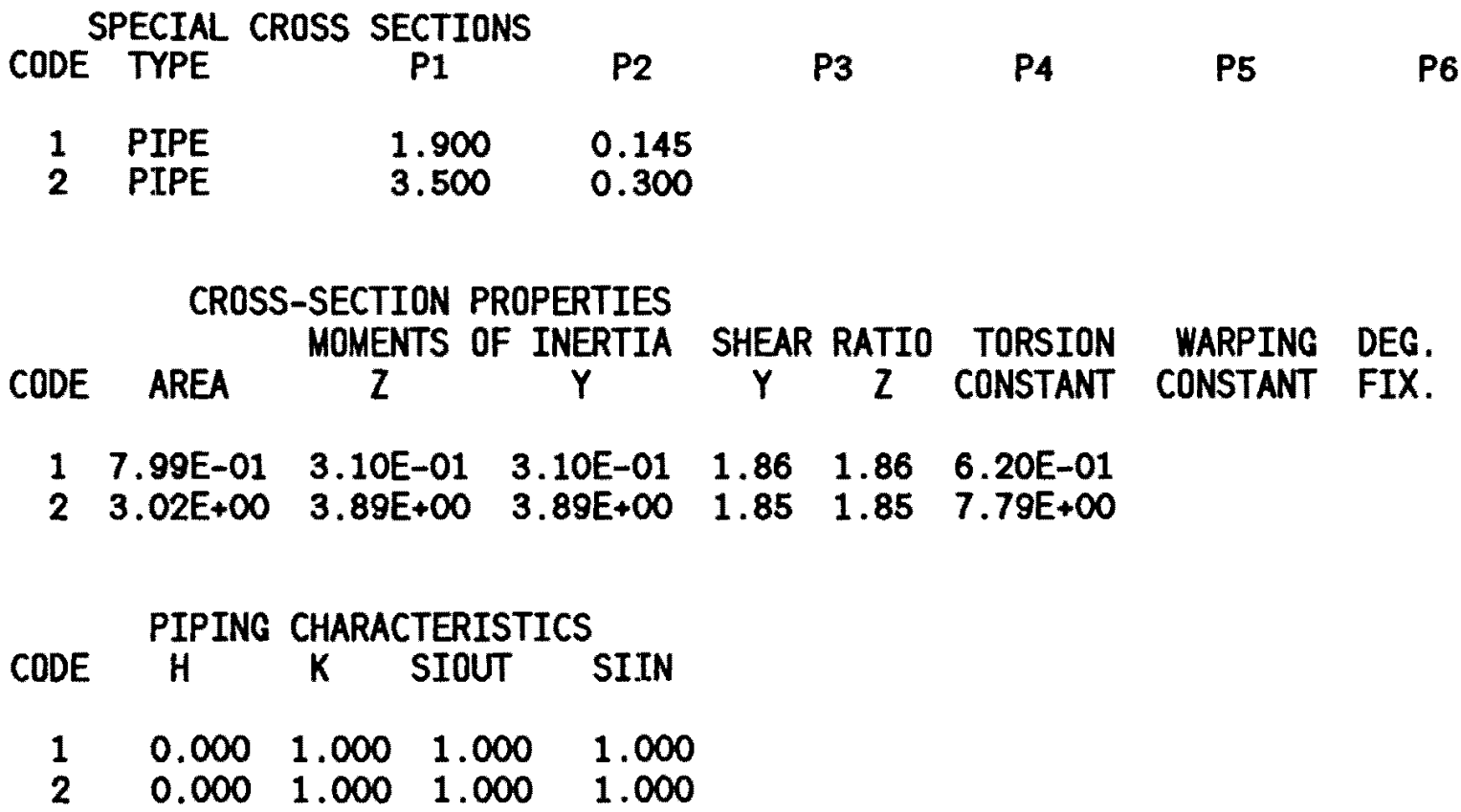

TOTAL STRUCTURE WEIGHT/MASS $=5.605 E+\infty 0$

C.G. LOCATION: $X=4.875 \quad Y=-2.875 \quad Z=10.516$

SPECIFIED RESTRAINTS

JOINT DIRECTION VALUE

$1 \quad 123456$

$6 \quad 123456$

LOADING NO. 1: THERMAL

TEMPERATURE LOADING

REFERENCE TEMPERATURE $=77$.

AMBIENT TEMPERATURE $=-325$.

TOTAL APPLIED FORCES:

$$
F(X)=0.000 E+00 \quad F(Y)=0.000 E+00 \quad F(Z)=0.000 E+\infty
$$

MOMENTS ABOUT ORIGIN:

$$
M(X)=0.000 E+\infty \quad M(Y)=0.000 E+\infty \quad M(Z)=0.000 E+\infty
$$


STATIC ANALYSIS OF GENERAL STRUCTURES

FRAME PIPE ANALYSIS LAR DEWAR COIL FEED 2/6/90

LOADING NO. 2: SUSTAINED

ACCELERATION LOADING

$A(X)=0.000 E+\infty \quad A(Y)=-1.000 E+\infty \quad A(Z)=0.000 E+\infty$

TOTAL APPLIED FORCES:

$F(X)=0.000 E+\infty \quad F(Y)=-5.605 E+\infty \quad F(Z)=0.000 E+\infty 0$

MOMENTS ABOUT DRIGIN:

$$
M(X)=5.894 E+01 \quad M(Y)=0.000 E+00 \quad M(Z)=-2.733 E+01
$$


DEGREES OF FREEDOM $=$ MAXIMUM BANDWIDTH $=$ AVERAGE BANDWIDTH $=$ PROFILE SIZE =

NUMBER OF JOINTS $=\quad 6$

NUMBER OF ELEMENTS $=\quad 5$

MAXIMUM JOINT NUMBER $=\quad 6$

MAXIMUM ELEMENT NUMBER $=5$

DIAGONAL ELEMENTS: AVERAGE $=1.825 E+06$ SMALLEST $=1.694 E+04 \mathrm{ROW}$ 
S A G S

STATIC ANALYSIS OF GENERAL STRUCTURES

STRUCTURAL DYNAMICS RESEARCH CORPORATION

FRAME PIPE ANALYSIS LAR DEWAR COIL FEED 2/6/90

UNITS USED FOR RESULTS:

UNITS FOR DISPLACEMENT ARE: IN

UNITS FOR FORCE ARE: LB

UNITS FOR STRESS ARE: PSI

LOAD DESCRIPTION

$\begin{array}{ll}1 & \text { THERMAL } \\ 2 & \text { SUSTAINED }\end{array}$

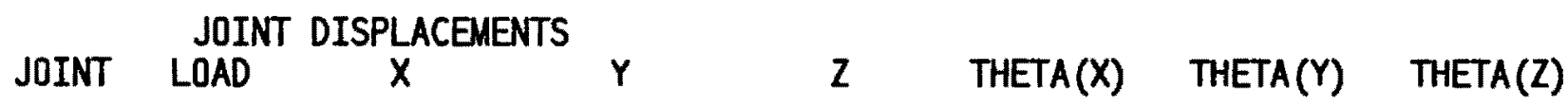

$2 \quad 1 \quad 5.256 \mathrm{E}-04 \quad 1.191 \mathrm{E}-02 \quad-2.009 \mathrm{E}-18 \quad 4.405 \mathrm{E}-19 \quad 9.049 \mathrm{E}-04 \quad 1.480 \mathrm{E}-04$

$2 \quad-5.347 \mathrm{E}-08-9.787 \mathrm{E}-07 \quad-4.155 \mathrm{E}-05 \quad 2.770 \mathrm{E}-05 \quad 2.570 \mathrm{E}-09-3.622 \mathrm{E}-08$

$3 \quad 1 \quad 1.933 \mathrm{E}-02 \quad 1.191 \mathrm{E}-02 \quad-5.854 \mathrm{E}-02 \quad-2.245 \mathrm{E}-19 \quad 1.286 \mathrm{E}-04 \quad 2.861 \mathrm{E}-06$

$2 \quad-6.628 \mathrm{E}-11 \quad-1.049 \mathrm{E}-03 \quad-4.155 \mathrm{E}-05 \quad 8.930 \mathrm{E}-05 \quad 3.650 \mathrm{E}-10 \quad-2.770 \mathrm{E}-07$

$4 \quad 1 \quad-1.933 \mathrm{E}-02 \quad 1.191 \mathrm{E}-02 \quad-5.854 \mathrm{E}-02 \quad-1.575 \mathrm{E}-19 \quad-1.286 \mathrm{E}-04 \quad-2.861 \mathrm{E}-06$

$2 \quad 4.126 \mathrm{E}-11-1.049 \mathrm{E}-03 \quad-4.155 \mathrm{E}-05 \quad 8.930 \mathrm{E}-05 \quad-3.652 \mathrm{E}-10 \quad 2.769 \mathrm{E}-07$

$5 \quad 1 \quad-5.256 \mathrm{E}-04 \quad 1.191 \mathrm{E}-02 \quad 4.471 \mathrm{E}-18$-1.034E-18 $-9.049 \mathrm{E}-04 \quad-1.480 \mathrm{E}-04$

$2 \quad 5.345 \mathrm{E}-08-9.787 \mathrm{E}-07 \quad-4.155 \mathrm{E}-05 \quad 2.770 \mathrm{E}-05 \quad-2.570 \mathrm{E}-09 \quad 3.621 \mathrm{E}-08$

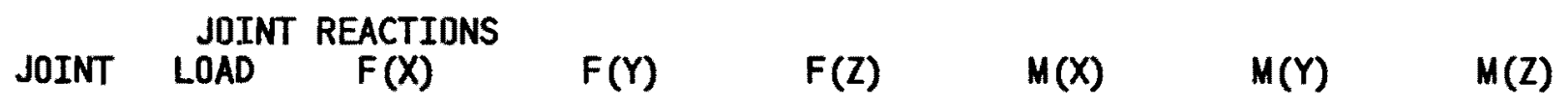

$1 \quad 1 \quad-1.206 E+02 \quad 4.547 \mathrm{E}-13 \quad 5.358 \mathrm{E}-13 \quad-1.272 \mathrm{E}-12 \quad-7.217 \mathrm{E}+02 \quad-3.384 \mathrm{E}+02$

$2 \quad-3.426 E-04 \quad 2.803 E+00-3.198 E-09-2.947 E+01 \quad-2.050 E-03 \quad 3.803 E-02$

$6 \quad 1 \quad 1.206 \mathrm{E}+02 \quad-4.547 \mathrm{E}-13 \quad-1.160 \mathrm{E}-12 \quad 2.840 \mathrm{E}-12 \quad 7.217 \mathrm{E}+02 \quad 3.384 \mathrm{E}+02$

$2 \quad 3.426 \mathrm{E}-04 \quad 2.803 \mathrm{E}+00 \quad 3.198 \mathrm{E}-09 \quad-2.947 \mathrm{E}+01 \quad 2.050 \mathrm{E}-03 \quad-3.801 \mathrm{E}-02$

LOAD JOINT REACTION TOTALS $F(X) \quad F(Y) \quad M(X) \quad M(Y) \quad M(Z)$

$\begin{array}{lllllll}1 & 0.000 E+\infty & 0.000 E+\infty & -6.241 E-13 & 1.568 E-12 & 0.000 E+\infty & 0.000 E+\infty\end{array}$

$2 \quad 0.000 E+00 \quad 5.605 E+\infty \quad 4.441 E-16 \quad-5.894 E+01 \quad 3.120 E-08 \quad 1.658 E-05$ 
STATIC ANALYSIS OF GENERAL STRUCTURES

FRAME PIPE ANALYSIS LAR DEMAR COIL FEED $2 / 6 / 90$

STRESS CALCULATIONS

\begin{tabular}{|c|c|c|c|c|c|c|c|c|}
\hline ЗEAM & LOAD & END & PT. & $\begin{array}{l}T \text { SHEAR } \\
P / A\end{array}$ & $\begin{array}{c}Y \text { SHEAR } \\
Y \text { BENDING }\end{array}$ & $\begin{array}{l}Z \text { SHEAR } \\
Z \text { BENDING }\end{array}$ & COMBINED & $\begin{array}{l}\text { STRESS } \\
\text { RATIO }\end{array}$ \\
\hline 1 & 2 & $\begin{array}{l}\text { FORE } \\
\text { AFT }\end{array}$ & 1 & $\begin{array}{l}1.106 \mathrm{E}+03 \\
5.688 \mathrm{E}-13 \\
3.142 \mathrm{E}-03 \\
3.215 \mathrm{E}+00\end{array}$ & $\begin{array}{l}2.813 E+02 \\
3.901 E-12 \\
7.990 E-04 \\
9.035 E+01\end{array}$ & $\begin{array}{r}-1.250 \mathrm{E}-12 \\
-1.037 \mathrm{E}+03 \\
7.457 \mathrm{E}-09 \\
1.197 \mathrm{E}-01\end{array}$ & $\begin{array}{l}2.444 E+03 \\
9.035 E+01\end{array}$ & $\begin{array}{l}0.13 \\
0.00\end{array}$ \\
\hline 2 & $\begin{array}{l}1 \\
2\end{array}$ & $\begin{array}{l}\text { AFT } \\
\text { FORE }\end{array}$ & 1 & $\begin{array}{r}3.608 \mathrm{E}+01 \\
-1.706 \mathrm{E}-12 \\
5.986 \mathrm{E}-02 \\
4.000 \mathrm{E}-09\end{array}$ & $\begin{array}{r}1.489 E-13 \\
-3.243 E+03 \\
-5.993 E+00 \\
6.284 E-03\end{array}$ & $\begin{array}{r}-2.813 E+02 \\
9.721 E-13 \\
-7.990 E-04 \\
9.035 E+01\end{array}$ & $\begin{array}{l}3.243 E+03 \\
9.035 E+01\end{array}$ & $\begin{array}{l}0.17 \\
0.00\end{array}$ \\
\hline 3 & $\begin{array}{l}1 \\
2\end{array}$ & $\begin{array}{l}\text { AFT } \\
\text { FORE }\end{array}$ & $\begin{array}{l}1 \\
1\end{array}$ & $\begin{array}{r}-4.637 E-14 \\
4.000 E+01 \\
9.860 E-08 \\
1.136 E-04\end{array}$ & $\begin{array}{r}5.338 E-14 \\
-4.753 E+02 \\
-8.737 E-01 \\
-1.350 E-03\end{array}$ & $\begin{array}{r}-2.155 E-13 \\
1.058 E+01 \\
1.959 E-09 \\
1.755 E-02\end{array}$ & $\begin{array}{l}4.754 E+02 \\
1.760 E-02\end{array}$ & $\begin{array}{l}0.03 \\
0.00\end{array}$ \\
\hline 4 & $\begin{array}{l}1 \\
2\end{array}$ & $\begin{array}{l}\text { FORE } \\
\text { AFT }\end{array}$ & 1 & $\begin{array}{r}-3.608 E+01 \\
1.138 E-12 \\
-5.984 E-02 \\
-4.000 E-09\end{array}$ & $\begin{array}{r}1.422 E-13 \\
-3.243 E+03 \\
5.993 E+00 \\
6.284 E-03\end{array}$ & $\begin{array}{r}2.813 E+02 \\
-7.823 E-13 \\
7.990 E-04 \\
9.035 E+01\end{array}$ & $\begin{array}{l}3.243 E+03 \\
9.035 E+01\end{array}$ & $\begin{array}{l}0.17 \\
0.00\end{array}$ \\
\hline 5 & $\begin{array}{l}1 \\
2\end{array}$ & $\begin{array}{l}\text { AFT } \\
\text { FORE }\end{array}$ & 1 & $\begin{array}{r}-1.106 E+03 \\
-5.688 E-13 \\
-3.142 E-03 \\
3.215 E+00\end{array}$ & $\begin{array}{r}-2.813 E \\
-8.707 E \\
-7.990 E \\
9.035 E\end{array}$ & $\begin{array}{r}-2.705 E-12 \\
-1.037 E+03 \\
7.457 E-09 \\
1.197 E-01\end{array}$ & $\begin{array}{l}2.444 E+03 \\
9.035 E+01\end{array}$ & 0.13 \\
\hline
\end{tabular}

S ORTED STRESS STRESS

BEAM LOC LOAD RATIO

$\begin{array}{llll}2 & 1.00 & 1 & 0.171 \\ 4 & 0.00 & 1 & 0.171 \\ 1 & 0.00 & 1 & 0.129 \\ 5 & 1.00 & 1 & 0.129 \\ 3 & 0.00 & 1 & 0.025\end{array}$


STATIC ANALYSIS OF GENERAL STRUCTURES

FRAME PIPE ANALYSIS LAR DEWAR COIL FEED $2 / 6 / 90$

\begin{tabular}{|c|c|c|c|c|c|}
\hline $\begin{array}{l}\text { ANSI } \\
\text { CASE }\end{array}$ & $\begin{array}{l}\text { B31. } 3 \mathrm{COI} \\
\text { THERMAL }\end{array}$ & $\begin{array}{l}\mathrm{E} \text { CHE } \\
\text { SUS? }\end{array}$ & $\begin{array}{l}\text { CKING } \\
\text { AINED }\end{array}$ & $\begin{array}{l}\text { CASES } \\
\text { DCCAS }\end{array}$ & SIONAL \\
\hline 1 & 1 & & 2 & & \\
\hline $\begin{array}{l}\text { ANSI } \\
\text { BEAM }\end{array}$ & $\begin{array}{l}\text { B31. } 3 \mathrm{CO} \\
\text { JOINT }\end{array}$ & $\begin{array}{l}\text { E CHE } \\
\text { CASE }\end{array}$ & $\begin{array}{l}\text { CK RES } \\
\text { STATU }\end{array}$ & $\begin{array}{l}\text { ULTS } \\
\text { IS EQ }\end{array}$ & RATIO \\
\hline $\begin{array}{l}1 \\
1\end{array}$ & $\begin{array}{l}1 \\
2\end{array}$ & $\begin{array}{l}1 \\
1\end{array}$ & $\begin{array}{l}\text { OK } \\
\text { OK }\end{array}$ & $\begin{array}{l}3 \\
3\end{array}$ & $\begin{array}{l}0.18 \\
0.16\end{array}$ \\
\hline $\begin{array}{l}2 \\
2\end{array}$ & $\begin{array}{l}2 \\
3\end{array}$ & $\begin{array}{l}1 \\
1\end{array}$ & $\begin{array}{l}\text { OK } \\
\text { OK }\end{array}$ & $\begin{array}{l}3 \\
3\end{array}$ & $\begin{array}{l}0.16 \\
0.23\end{array}$ \\
\hline $\begin{array}{l}3 \\
3\end{array}$ & $\begin{array}{l}3 \\
4\end{array}$ & $\begin{array}{l}1 \\
1\end{array}$ & $\begin{array}{l}\text { OK } \\
\text { OK }\end{array}$ & $\begin{array}{l}1 \\
1\end{array}$ & $\begin{array}{l}0.07 \\
0.07\end{array}$ \\
\hline $\begin{array}{l}4 \\
4\end{array}$ & $\begin{array}{l}4 \\
5\end{array}$ & $\begin{array}{l}1 \\
1\end{array}$ & $\begin{array}{l}\text { OK } \\
\text { OK }\end{array}$ & $\begin{array}{l}3 \\
3\end{array}$ & $\begin{array}{l}0.23 \\
0.16\end{array}$ \\
\hline $\begin{array}{l}5 \\
5\end{array}$ & $\begin{array}{l}5 \\
6\end{array}$ & $\begin{array}{l}1 \\
1\end{array}$ & $\begin{array}{l}\text { OK } \\
\text { OK }\end{array}$ & $\begin{array}{l}3 \\
3\end{array}$ & $\begin{array}{l}0.16 \\
0.18\end{array}$ \\
\hline
\end{tabular}

MAXIMLM RATIO $=0.23$ ON BEAM 2 
STATIC ANALYSIS OF GENERAL STRUCTURES

FRAME PIPE ANALYSIS LAR DEWAR COIL FEED 2/6/90

TABLE OF CONTENTS

PAGE

OUTPUT BY JOINT AND ELEMENT

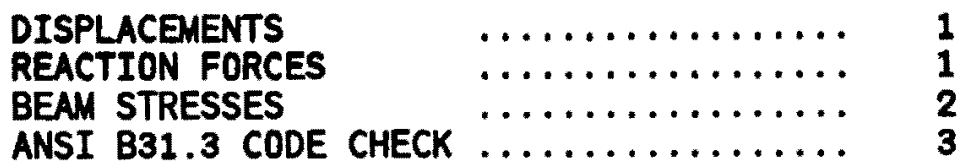


SDRC 1-DEAS 4.1: Frame Analysis
(-D

DATABASE: LAR STOR. DEWAR LN2 COIL FEED LINE (1/31/9)

VIEW : No stored VIEW

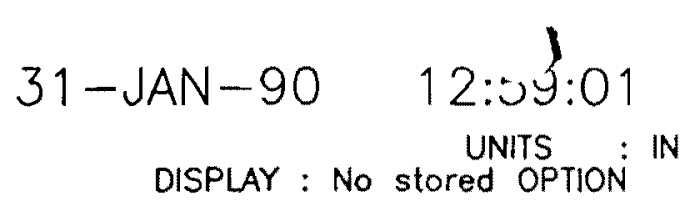

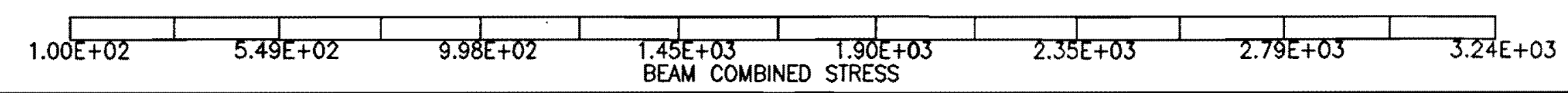

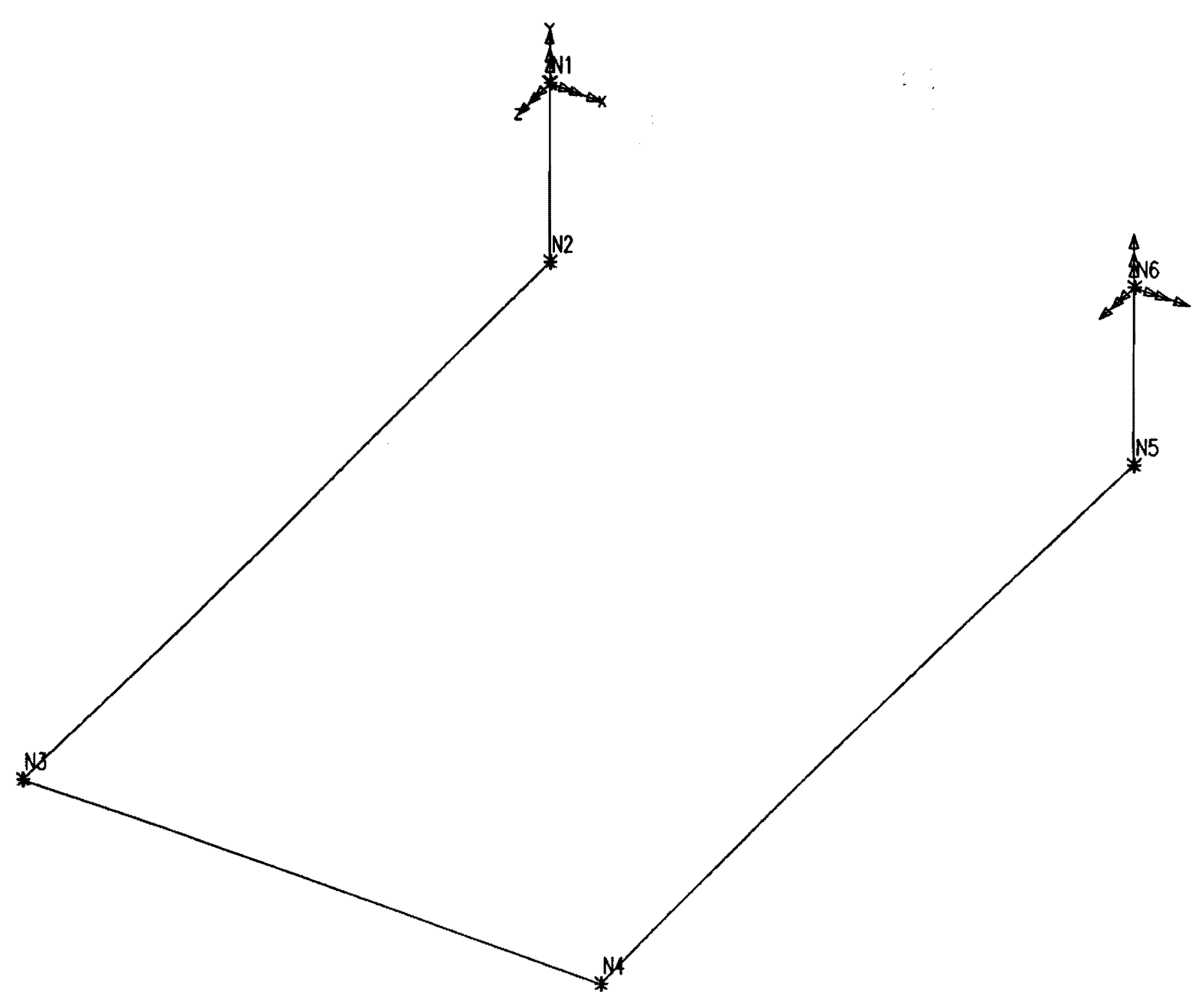


'SDRC I-DEAS 4.1: Frame Analysis')

LAR STOR. DEWAR LN2 COIL FEED LINE $(1 / 31 / 9)$

VIEW : No stored VIEW

FRAME PIPE ANALYSIS LAR DEWAR COIL FEED $1 / 31 / 90$
31-JAN-90 12:E ?46 UISPLAY : No stored OPTION

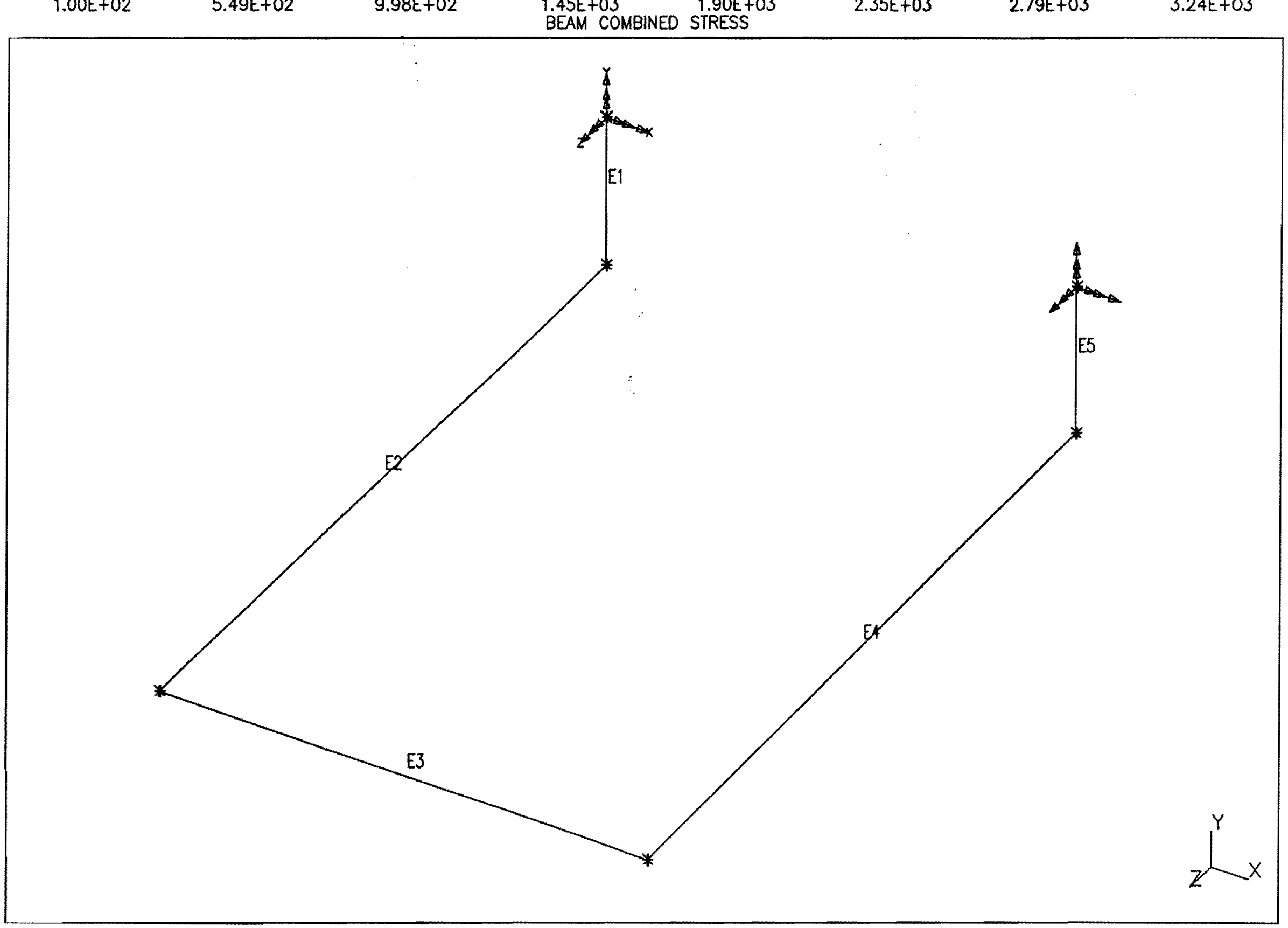


) SDRC I-DEAS 4.1: Frame Analysis' DATABASE: LAR STORAGE DEWAR COIL FEED LINES $(2 / 6 / 90)$ VIEW : NO StOred VIEW

\section{6-FEB-90 09:40:13}

DISPLAY : No stored OPTION :

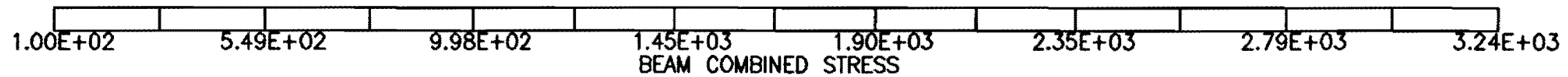

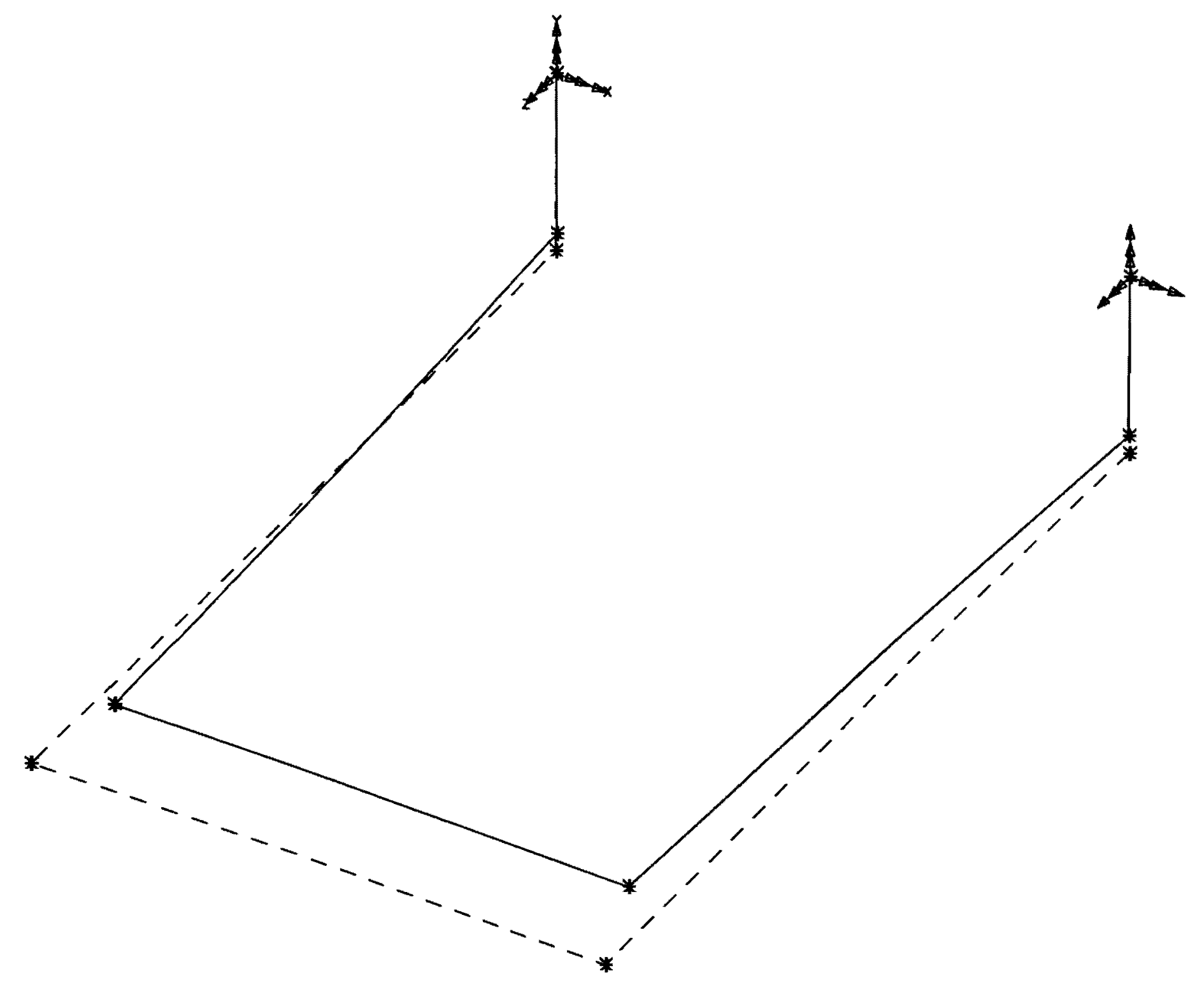

$L^{Y} x$ 


\section{Appendix A}

1. PEI drawing C-32545, Rev 0 see feed lines, item 5 .

2. Scott C. Nason, PEI engineer, 11/20/89 letter see items 1,2 , and 3 . 
November 20,1989

FERMI NATIONAL ACCELERATOR LABORATORY

Batavia Rd.; P.0, Box 500

Mail Station 357

Batavia, 111 inois 60510

Attention: Mr. George Mulholland

Subject: Fermi P.0. No. 918320

Model 20000-H-65 Liquid Argon Storage Tank

PEl Job No. C-17986/N=03687

Dear George:

To address your recent questions on the subject unit:

1. The internal coil piping material is $1-1 / 2^{*}$ schedule 40 (.145" wall), type $\mathbf{5 0 8 3}$ or $\mathbf{5 0 8 6}$ aluminum. The headers of the coil are $3^{\prime}$ schedule $80\left(.300^{*}\right.$ wall) type $\mathbf{5 0 8 6}$ aluminum.

2. The coil feed lines are 1-1/2" schedule $10\left(.109^{\prime}\right.$ wall) type 304 stainless steel (welded pipe), not 1-5/8" ODT as indicated on our customer drawing. 200 psi.

3. The internal coil, including the feed lines, was pneumatic tested at

4. The liquid level chart for this unit will not have the same 'trap error" as was found on the nitragen dewar ( $C-28087, N-64587$ ) because the bottom gage 1 ine on this unit properly exits the annular space at the elevation of the bottom of the inner vessel. We believe the error in the layout of the bottom gage line on the nitrogen dewar was an isolated one.

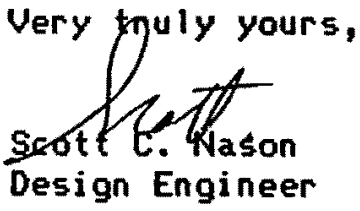

cc: Mr. Kenneth L. Paul, Executive Vice President, PEI

PEI File C-85037

Hand-delivered, $11 / 20 / 89$, at PEI 